\title{
SEPPELLIRE UN VESCOVO, SEPPELLIRE UN MONACO. LA GESTIONE DELLA MORTE ALL'INTERNO DEL COMPLESSO DI SAN SEVERO IN CLASSE A RAVENNA
}

\section{DEBORA FERRERI}

UDC: $27-788(450.457)$ 27-55

Review

Manuscript received: 04. 11. 2016.

Revised manuscript accepted: 23. 01. 2017.

DOI: 10.1484/J.HAM.5.113752
D. Ferreri

Università di Bologna Scuola di scienze Science for Conservation-Restoration of Cultural Heritage Via Guaccimanni Ravenna

Italia

The complex of San Severo in Classe, in south of Ravenna, is an emblematic place of memory to understand the funerary practice in this area. The monastery was built in the late ninth century, close to the basilica and to the mausoleum dedicated to one of the most important bishops of Ravenna, who lived in the fourth century. The political and religious importance of the monastic center is attested by written sources since the tenth century. The monastery was a Benedectine foundation, which passed to the Cistercensian between the twelfth and thirteenth centuries. The current excavation are being conducted by the University of Bologna, which is investigating extensively the entire complex, bringing to light the different areas of the monastery around the large rectangular cloister. The funerary practice changed and developed according to the life of the basilica and the monastic complex. Different funerary areas found outside the church and in the monastery have different characteristics and may be linked to different chronological phases as well as to different functions or perceptions of the areas themselves. The organization of the monastery cemetery indicating funerary spaces for member of monastic community and clergy, separate from those of the laity.

Keywords: Monastery, burials, death, memory building, Ravenna

Il complesso di San Severo si trova oggi nel suburbio meridionale di Ravenna. A partire dal 2006 fino al 2016 il sito è stato oggetto delle indagini dell'Università di Bologna, attraverso estensive campagne archeologiche che hanno portato alla luce la basilica, dedicata a uno dei primi vescovi di Ravenna, Severo, e un grande monastero benedettino edificato alla fine del IX secolo. Si tratta dell'unico monastero in area ravennate indagato archeologicamente per tutta la sua estensione ${ }^{1}$.

La lunga diacronia del sito, che va dall'età romana al pieno Medioevo, offrendo un caso molto importante per conoscere le dinamiche insediative e la cultura materiale in un arco di tempo così vasto, permette anche di analizzare la pratica funeraria e la gestione della memoria in questo territorio, cogliendo soprattutto le trasformazioni e i cambiamenti del rapporto con la morte, e il modo in cui essa viene gestita ${ }^{2}$. Il complesso, situato nei pressi di una necropoli romana3, è costruito in parte sui resti di una grande villa suburbana di tarda età repubblicana, come altre rinvenute nell'area di Classe, e utilizzata, con molte trasformazioni, fino agli inizi del VI secolo, seguendo alcune dinamiche tra necropoli e complessi residenziali antichi analizzate e localizzati in diverse regioni italiane ${ }^{4}$.

In questo stesso luogo, verso la metà del IV secolo, su parte degli ambienti termali dell'edificio residenziale, è stato costruito un piccolo mausoleo absidato, con un ingresso a trifora, in cui, secondo lo storico Agnello, venne seppellito Severo, vescovo di Ravenna, vissuto nella metà del IV secolo5.

\footnotetext{
${ }^{1}$ Le indagini archeologiche sono dirette dal prof. Andrea Augenti e coordinate dal dott. Enrico Cirelli, in un progetto che ha visto la collaborazione dell'Università di Bologna e della Fondazione Ravennantica. Al progetto hanno partecipato numerosi studenti e ricercatori di diverse università italiane e straniere. ${ }^{2}$ L'analisi delle pratiche funerarie a Classe attraverso il complesso di San Severo e l'area portuale sono state oggetto del mio progetto di Dottorato conseguito presso l'Università di Siena dal titolo "Archeologia funeraria a Classe tra la tarda antichità e il Medioevo: il complesso di San Severo e l'area portuale", tutor prof. Andrea Augenti.

${ }_{3}^{3}$ Per la necropoli romana: G. CORTESI, La zona e la basilica di S. Severo nel territorio di Classe, Ravenna, 1964; G. CORTESI, Classe paleocristiana e bizantina, Ravenna, 1980. Per i materiali rinvenuti G. BERMOND MONTANARI, Introduzione ai problemi relativi alle necropoli di età romana di Ravenna e Classe, in CARB, 35, 1988, p. 237-242; M.G. MAIOLI, M.L. STOPPIONI, Classe e Ravenna tra terra e mare: città, necropoli, monumenti, Ravenna, 1987. L'esistenza di una zona cimiteriale precedente alla costruzione della basilica è testimoniata anche da Andrea Agnello nella Vita di Giovanni II (v. nota 5) Agnello XII; XLI, V. ${ }_{4}^{4}$ Per la villa al di sotto della basilica e nel territorio di Classe si rimanda a G. BERMOND MONTANARI, La chiesa di S. Severo nel territorio di Classe. Risultati dei recenti scavi, Bologna, 1968, p. 63-65; M.G. Maioli, La topografia nella zona di Classe, in Susini G. (a cura di), Storia di Ravenna, I. L'evo antico. Venezia, 1990, p. 375-414; M. G. MAIOLI, Nuovi dati sul complesso archeologico di San Severo a Classe (RA): scavi 1981-1991, in CARB, 39, 1992, p. 497-520; J. ORTALLI, L'edilizi abitativa, in A. Carile (a cura di), Storia di Ravenna, II, 1. Dall'età bizantina all'età ottoniana, Venezia, 1991, p. 179-181; A. Augenti (a cura di), Classe. Indagini sul potenziale archeologico di una città scomparsa, Bologna, 2011. Il tema dell'evoluzione delle ville e la continuità di uso di queste strutture è oggetto di numerose ricerche e studi. La bibliografia è molto ampia; per una sintesi si rimanda a G.P. Brogiolo (a cura di), La fine delle ville romane: trasformazioni nelle campagne tra tarda antichità e alto medioevo, Mantova, 1996 (Documenti di archeologia, 11); R. FRANCOVICH, R. HODGES, From Villa to Village. The Transformation of the Roman Countryside in Italy, c. 400-100o, London, 2003; G.P. Brogiolo, A. Chavarría Arnau, M. Valenti (a cura di), Dopo la fine delle ville: le campagne dal 6. al 9. secolo, Seminario sul tardo antico e l'alto Medioevo (Gavi, 8-10 maggio 2004), Mantova, 2005; G. P. BROGIOLO, A. CHAVARRÍA ARNAU, Aristocrazie e campagne nell'Occidente da Costantino a Carlo Magno, Firenze, 2005.

${ }^{5}$ Riguardo la vita del vescovo si rimanda al Codex Pontificalis Ecclesiae Ravennatis di Andrea Agnello in A. Testi Rasponi (a cura di), Agnelli Liber Pontificalis, in Rerum Italicarum scriptores, II, 3, Bologna, 1924, p. 71; M. MAZZOTTI, San Severo di Ravenna e la basilica a lui dedicata nel territorio di Classe, in CARB, 15, 1968, p. 230. Il racconto della sua morte è in Andrea Agnello, XII, p. 49, la vita del santo è scritta dal monaco tedesco Liutolfo: L. De Heinemann (ed.), Liutolfus, Vita et translatio S. Severi, in Monumenta Germaniae Historica, XV, 1, Hannoverae, 1887, p. 289-293. Con il passare del tempo il racconto della morte divenne sempre più grandioso, arrivando a immaginarlo addirittura all'interno della basilica stessa, ignorando completamente la storia del monumento ravennate. A proposito si veda: P. NATALI, Catalogus sanctorum, Liber Tertius, f. XLVII, cap. LXV, 1534, e G. RIPOLL, Il santo, le reliquie, in
} 


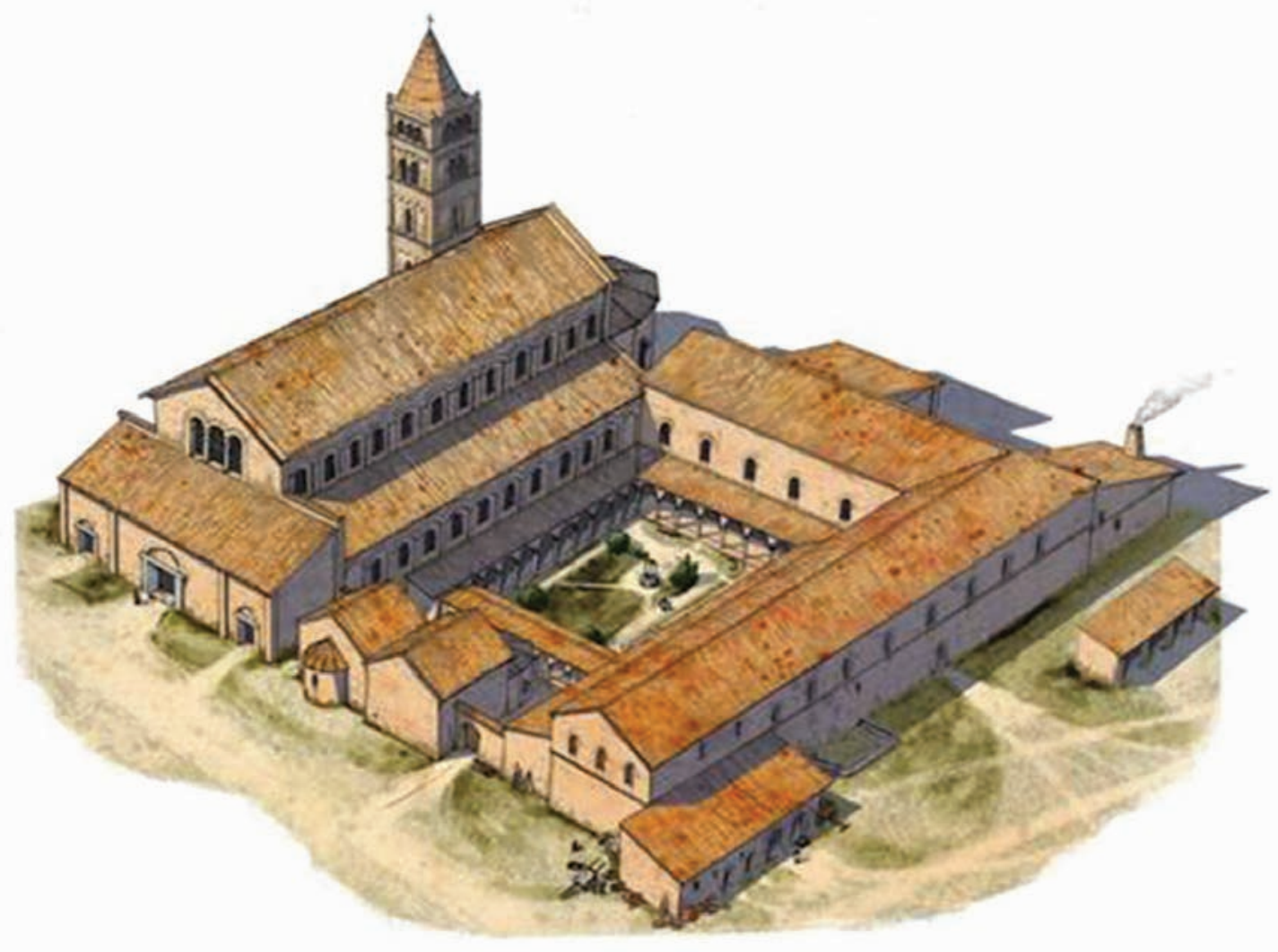

FIG. 1: Ricostruzione del complesso di San Severo (disegno di G. Albertini).

A partire dal $\mathrm{V}$ secolo questa zona fu inglobata all'interno delle mura della città, trasformando l'area da suburbana in urbana ${ }^{6}$, e modificando in questo modo il rapporto del sito con il territorio circostante. Successivamente, intorno al 58 o d.C., accanto a questo mausoleo venne edificata una monumentale basilica destinata alla commemorazione della memoria del vescovo, intorno al quale furono disposte numerose sepolture, attratte dal culto del santo ${ }^{7}$, con forme semplici o elaborate, a seconda del periodo e dell'organizzazione cimiteriale, che muta nel corso del tempo ${ }^{8}$.
La basilica, sia nell'aspetto monumentale sia nella sua storia, è uno dei più importanti luoghi di culto dell'area ravennate, elemento trainante nella costruzione dell'identità comunitaria. Il complesso rappresenta l'ultimo capitolo di una straordinaria stagione edilizia che ha interessato Ravenna e Classe e forse il canto del cigno della committenza arcivescovile nell'antica sede imperiale e ora capitale esarcale9. La costruzione dell'identità della popolazione di Classe ha una base importante nella progettazione e nella costruzione dei luoghi destinati al culto dei vescovi e dei

\footnotetext{
A. Augenti (a cura di), La basilica e il monastero di San Severo. La storia, gli scavi, Ravenna, 2006, p. 9-11. Sulle recenti indagini archeologiche di questo edificio si rimanda a D. FERRERI, La città dei vivi e la città dei morti. La ridefinizione degli spazi urbani e delle pratiche funerarie a Ravenna e nel territorio circostante tra la tarda Antichità e l'alto Medioevo, in Hortus Artium Medievalium, 20, 2014, p. 112-123.

${ }^{6}$ Sulle indagini relative alle mura di Classe si veda G. LEPORE, G. MONTEVECCHI, Classe (Ravenna), Suburbio, in Ocnus, 17, 2009 , p. $155-157$.

${ }_{7}$ Circa i mausolei cristiani si vedano: G.P. BROGIOLO, Oratori funerari tra VII e VIII secolo nelle campagne transpadane, in Hortus Artium Medievalium, 8, 2002, p. 9-31; A. CHAVARRÍA ARNAU, Splendida sepulcra ut posteri audient. Aristocrazie, mausolei, e chiese funerarie nelle campagne tardo antiche, in G.P. Brogiolo, A. Chavarria Arnau (a cura di), Archeologia e società tra tardo antico e alto Medioevo, Mantova, 2007, p. 127-146; G.P. BROGIOLO, G. CANTINO WATAGHIN, S. GELICHI, L'Italia settentrionale, in P. Pergola (a cura di), Alle origini della parrocchia rurale, Atti della giornata tematica dei Seminari Archeologia Cristiana (École française de Rome, 19 marzo 1998), Città del Vaticano, 1999.

${ }^{8}$ Per un'analisi delle sepolture e degli spazi cimiteriali relativi alla basilica di San Severo: D. FERRERI, Sepolture e riti funerari a Classe: una lunga prospettiva diacronica, in G. Volpe, P. Favia (a cura di), V Congresso Nazionale di Archeologia Medievale (Foggia-Manfredonia, 30 settembre-3 ottobre 2009), Firenze, 2009, p. 459-464; D. FERRERI, Spazi cimiteriali, pratiche funerarie e identità nella città di Classe, in Archeologia medievale, 38, 2011, p. 59-74; I. BARBIERA, D. FERRERI, Placing bodies and constructing memory at San Severo, in Rasson J., Szende K. (eds.), in Annual of Medieval Studies, 13, Budapest, 2007, p. 187-196. ${ }_{9}$ A. AUGENTI, Nascita e sviluppo di una capitale: Ravenna nel V secolo, in P. Delogu, S. Gasparri (a cura di), Le trasformazioni del V secolo. L'Italia, $i$ barbari e l'occidente romano, Atti del seminario di Poggibonsi (18-20 ottobre 2007), Turnhout, 2010, p. 343-369; A. AUGENTI, Immaginare una comunità, costruire una tradizione. Aristocrazie e paesaggio sociale a Ravenna tra V e X secolo, in G.P. Brogiolo, A. Chavarría Arnau (a cura di), Archeologia e società tra tardo antico e alto medioevo, Atti del $12^{\circ}$ seminario sul tardo antico e l'alto medioevo (Padova, 29 settembre - 1 ottobre 2005), Mantova, 2007, p. 193-204. ${ }^{10} \mathrm{La}$ tomba dei martiri e la presenza di reliquie dei santi erano elementi di attrazione sia delle sepolture sia della popolazione attorno agli edifici religiosi. Questo avveniva per le reliquie persino quando non erano noti esattamente quali santi ci fossero conservati o le modalità e i tempi di ottenimento di queste da parte delle chiese (D. NUZZO, Reliquie ed edifici di culto rurali nel V-VII secolo, in A. Coscarella, P. De Santis (a cura di), Martiri, santi, patroni: per un'archeologia della devozione, Atti X Congresso Nazionale di Archeologia Cristiana (Università della Calabria, 15-18 settembre 2010), Rossano (Cs), 2012, p. 329-346. Sulle sepolture e gli edifici religiosi si rimanda inoltre a C. SAPIN, C. TREFFORT, Inhumations et edifices religieux au Moyen Âge. Bilan d'une rencontre et pistes de réflexion, in A. Alducle Bagousse (a cura di), Inhumations et edifices religieux au Moyen Âge entre Loire et Seine, Crahm, 2004, p. 3-12; C. SAPIN, Construire et fonder une mémoire, in I. Cartron, Barraud, D. P. Henriet, A. Michel (eds.), Autour de Saint-Seurin: lieu, mémoire, pouvoir des premiers temps chrétiens à la fin du moyen âge, Actes du Colloque (Bordeaux, 12-14 octobre 2006), Bordeaux, 2009, p. 173-175.
} 
santi locali ${ }^{10}$. L'edificio, in parte scavato negli anni '6o, ha caratteristiche architettoniche simili alla vicina e poco anteriore basilica di Sant'Apollinare che, a differenza della basilica di San Severo, era posta al di fuori delle mura della città di Classe ${ }^{11}$.

Alla fine del IX secolo accanto alla basilica, sul lato meridionale dell'edificio, viene costruito un monastero benedettino, menzionato in un atto notarile del $955^{12}$. I reperti ceramici hanno permesso di datare alla seconda metà del IX secolo la sua edificazione, anticipandola di oltre un cinquantennio rispetto alle fonti scritte ${ }^{13}$. Di poco successivi sono alcuni documenti, i quali attestano un importante evento che avvenne in questo luogo nell'anno 967: Ottone I risedette in una mansio domnicata presso il monastero e, all'interno dell'edificio, tenne un importante placito alla presenza del papa, dell'arcivescovo di Ravenna e di diversi esponenti delle aristocrazie europee della metà del X secolo ${ }^{14}$ (fig. 1).

Lo sviluppo del monachesimo a Ravenna e nel territorio circostante è un fenomeno ben studiato dal punto di vista delle ricerche storiche, ma non archeologiche ${ }^{15}$. Sebbene di alcuni monasteri si conservino le tracce, come S. Apollinare in Classe, S. Apollinare Nuovo, S. Stefano in Fondamento regis e S. Maria in Coeloseo, quello di San Severo è l'unico indagato in maniera estensiva e con una ricerca programmatica $^{16}$.

\section{IL MONASTERO DI SAN SEVERO: LE INDAGINI ARCHEOLOGICHE}

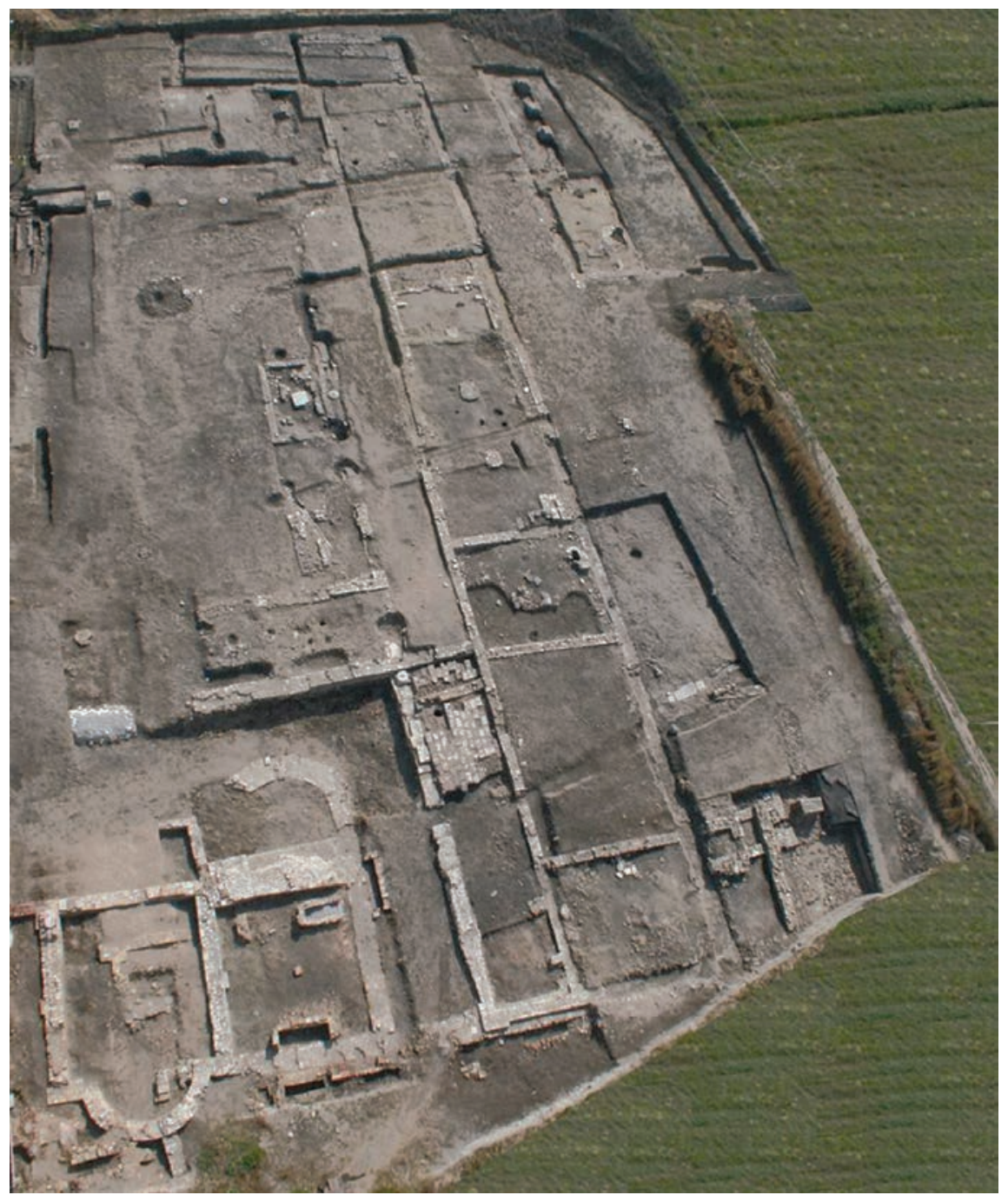

FIG.2 : Immagine dall'alto del monastero in corso di scavo (foto di E. Cirelli)
Il sito si sviluppa su di un'area di circa $2500 \mathrm{mq}$, che si estende lungo tutto il muro perimetrale sud della basilica, e con un lato costituito dall'area del sacello. Di alcuni degli ambienti indagati è stato possibile interpretare la funzione, come la sala capitolare, lo scriptorium, il refettorio, le cucine, le aree produttive; altri vani e zone del monastero sono in corso di indagine e definizione ${ }^{17}$. Le trasformazioni architettoniche più significative del monastero avvengono in un periodo compreso tra la fine del IX e gli inizi del X secolo, come hanno dimostrato le evidenze materiali ${ }^{18}$ (fig. 2).

Tra la grande basilica e gli ambienti monastici si trova un grande chiostro altomedievale, costruito nella prima fase del

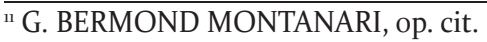

${ }^{12}$ R. BENERICETTI, Le Carte ravennati del X secolo. Archivio Arcivescovile II (aa. 900-955), Faenza, 1999, p. 207, 210. Un'altra menzione del monastero di San Severo risale al 959, attestata in V. BUZZI, La curia arcivescovile e la curia cittadina di Ravenna dall'850 al 1118, in Bullettino dell'Istituto Storico Italiano per il Medieovo e Archivio Muratoriano, 35, 1915, p. 9, 38; B. CAVARRA, G. GARDINI, G.B. PARENTE, G. VESPIGNANI, Gli archivi come fonti per la storia di Ravenna, in A. Carile (a cura di), Storia di Ravenna, II. Dall'età bizantina all'età ottoniana, 1. Territorio, economia, società, Venezia, 1991, p. 401-547; M. FANTUZZI, Monumenti Ravennati de secoli di Mezzo per la maggior parte inediti, Venezia, 1801-1804.

${ }^{13}$ E. CIRELLI, E. LO MELE, La cultura materiale di San Severo alla luce delle nuove scoperte archeologiche, in P. Racagni (a cura di), La Basilica ritrovata. I restauri dei mosaici antichi di San Severo a Classe, Ravenna, Bologna, 2010, p. 39-57.

${ }^{14}$ B. CAVARRA, G. GARDINI, G. B. PARENTE, G. VESPIGNANI, op. cit., p. 479-48o; M. FANTUZZI, op. cit., p. 27-30; R. BENERICETTI, Le carte ravennati del X secolo: Archivio vescovile, 976-999, Imola, Bologna, 2002.

${ }^{15}$ P. NOVARA, I monasteri ravennati in età ottoniana: una difficile indagine archeologica, in P. Novara (a cura di), Ad religionis claustrum construendum: monasteri nel medioevo ravennate: storia e archeologia, Ravenna, 2013.

${ }^{16}$ E. CIRELLI, Ravenna: archeologia di una città, Firenze, 2008.

${ }^{17}$ Per una prima analisi del complesso monastico di San Severo si veda: A. AUGENTI, E. CIRELLI, San Severo and religious life in Ravenna during the ninth and tenth centuries, in J. Herrin and J. Nelson (ed.), Ravenna: its role in earlier medieval change and exchange, London, 2016, p. 297-321; A. AUGENTI, I. BEGNOZZI, M. BONDI, E. CIRELLI, D. FERRERI, C. MALAGUTI, P. SCOZZARI, Il monastero di San Severo a Classe: risultati delle campagne di scavo 2006-2011, in F. Redi, A Forgione (a cura di), Atti del VI convegno SAMI (L'Aquila 2012), Firenze, 2012, p. 238-245. Sui monasteri in Europa occidentale si veda F. DE RUBEIS, F. MARRAZZI, Monasteri in Europa occidentale (VIII-IX): topografia e strutture, Atti del Convegno Internazionale, Castel San Vincenzo 2004, Roma, 2008.
}

${ }^{18}$ E. CIRELLI, E. LO MELE, op. cit. (nota 13), p. 41. 


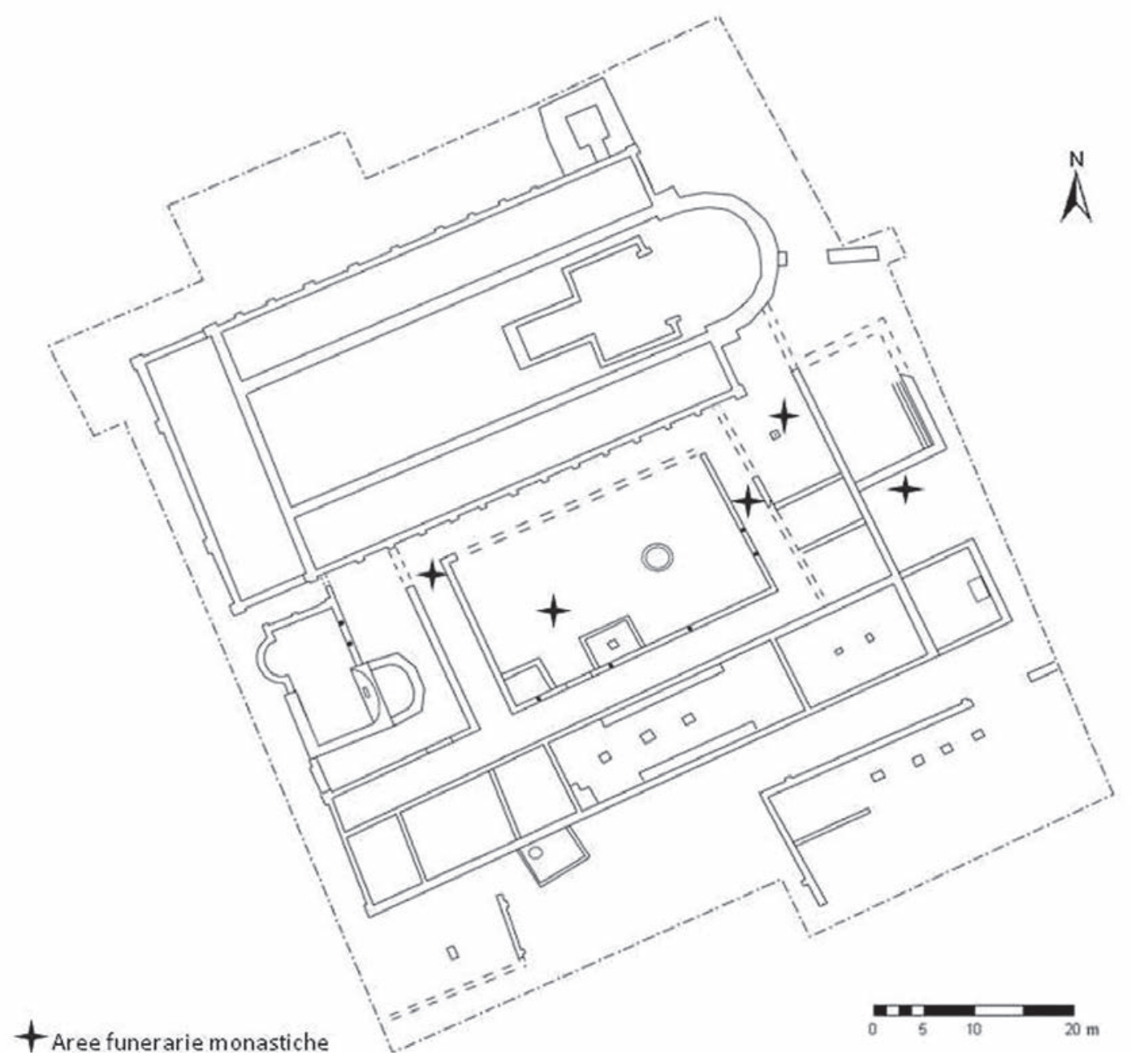

FIG.3: Pianta del complesso di San Severo. In evidenza le aree cimiteriale del monastero.

monastero, circondato da un portico monumentale, caratterizzato dalla compresenza di colonne e pilastri sul lato sud, e da sole colonne sul lato ovest. Di queste colonne, asportate nella seconda metà del XV secolo, restano in situ le basi, di circa $53 \mathrm{~cm}$ di diametro, risparmiate solo perché coperte dall'innalzamento dei piani pavimentali ${ }^{19}$. Sul lato nord della chiesa, invece, viene costruito un campanile a pianta quadrata, nelle cui fondazioni è stato individuato materiale che ne consente la datazione tra la fine dell'XI e il XII secolo. La costruzione del campanile oblitera l'ambiente addossato nel VII secolo a nord-est della basilica ${ }^{20}$. Per la realizzazione del complesso vengono impiegati soprattutto materiali di recupero provenienti dall'edificio romano sottostante e probabilmente da altri edifici, in stato di abbandono, della città di Classe.

Le aree funerarie più antiche, situate presso il mausoleo e la basilica, continuano a essere utilizzate anche dopo la costruzione dell'edificio monastico, incrementando e inten- sificando la densità delle sepolture, e in alcuni casi apportando delle novità nell'organizzazione cimiteriale, con aree destinate a gruppi e nuclei di sepolture, mentre altre sono destinate a tombe multiple e familiari, come rilevato dalle analisi antropologiche effettuate sui resti scheletrici ${ }^{21}$. Negli ambienti prettamente monastici sono state ritrovate, invece, numerose sepolture appartenenti ai membri della comunità monastica, in accordo con quanto evidenziato dalle testimonianze scritte che indicano l'uso di seppellire i monaci in spazi cimiteriali appositi, distinti rispetto a quelli dei laici ${ }^{22}$.

La riforma ecclesiastica carolingia, che ebbe il suo culmine tra IX eX secolo, apportò diversi cambiamenti al sistema funerario, attuando una legislazione più rigida riguardo agli spazi cimiteriali eal rituale, con la gestione dei cimiteri e della pratica sepolcrale da parte del clero. I capitolari carolingi ribadirono questo principio più volte, segno della volontà ecclesiastica di sovrintendere la nuova polarizzazione dei centri di culto e gestire la spiritualità dei fedeli per avere un

\footnotetext{
${ }^{19}$ Nelle ultime fasi di vita il complesso viene spoliato dei materiali edilizi che saranno reimpiegati per la costruzione di altri edifici, tra cui il tempio malatestiano di Rimini. A tal proposito si veda C. BERTELLI, Ravenna e l'Infelix Italia, in A. Augenti, C. Bertelli (a cura di), Felix Ravenna. La croce, la spada, la vela: l'alto Adriatico fra Ve VI secolo, Catalogo della mostra (Ravenna, 2007), Milano, 2007, p. 19-26.

${ }^{20}$ N. CHRISTIE, G. MUSINA, G. SPEED, Settore 10 (1000o). Annesso nord, in A. Augenti, N. Christie, J. Laszlowsky, G. Ripoll (eds.), San Severo (Classe, Ravenna). Relazione finale degli scavi archeologici (Campagna 2006), Bologna, 2017 in corso di stampa, p. 133-142.

${ }^{21}$ D. FERRERI, op. cit. (nota 8).

${ }^{22}$ Per gli spazi funerari nei monasteri si veda G. CANTINO WATAGHIN, E. DESTEFANIS, Les espace funéraires dans les ensembles monastiques du Haut Moyen Âge, in M. Lauwers (ed.), Monastères et espace social. Genèse et transformation d'un système de lieux dans l'Occident médiéval, Collection d'études médiévales de Nice, 15, Turnhout, 2014, p. 503-553; R. GILCHRIST, Monastic and Church Archaeology, in Annual Review of Anthropology, 43, 2014, p. 235250; R. GILCHRIST, B. SLOANE, Requiem: The Medieval monastic cemetery in Britain, London, 2005.
} 
maggiore potere su di loro anche controllandone le pratiche funerarie ${ }^{23}$.

Tutto questo non trovò facile attuazione, viste le profonde ingerenze di potenti laici nell'esercizio della fede di coloro che frequentavano le loro chiese o i monasteri privati ${ }^{24}$. La Chiesa amministrava il diritto di sepoltura e anche l'attività di commemorazione e di gestione della memoria del defunto. Dopo l'intervento carolingio i monaci amministravano le attività cimiteriali, $\mathrm{i}$ beni donati in punto di morte, e anche le commemorazioni successive. Nel 1128 l'arcivescovo Gualtiero, ad esempio, conferma in perpetuo a Divizio, abate di S. Severo, gli antichi privilegi inerenti ai beni del suo monastero, con l'impegno però da parte dei monaci di cantare messe con 50 salmi ogni anniversario della sua morte ${ }^{25}$.

Le aree cimiteriali dei monasteri sono generalmente caratterizzate da una densità di sepolture minore rispetto ai cimiteri parrocchiali. In alcuni casi è la conseguenza di una restrizione dello spazio funerario. La riforma carolingia infatti, conferì ai soli religiosi la possibilità di essere sepolti all'interno dei complessi monastici, escludendo le sepolture dei laici dallo spazio claustrale ${ }^{26}$. Sebbene la norma benedettina, redatta attorno agli anni 530-540, non indichi particolari necessità di disporre di un luogo specifico dove inumare i defunti, in molti monasteri sono attestate aree funerarie differenziate. Mentre gli spazi collegati alla basilica, sia il suo interno sia le aree esterne che la circondano, sono accessibili ai laici, gli spazi del monastero sono a uso esclusivo per i monaci o per i membri che vivevano all'interno del monastero.

Nella maggior parte dei casi le evidenze archeologiche rivelano due possibili nuclei cimiteriali nei monasteri: la sala capitolare e lo spazio del chiostro, comprendendo il cortile o i corridoi laterali che lo circondavano ${ }^{27}$ (fig. 3).

La gestione della pratica funeraria da parte del monastero di San Severo è attestata anche dalle fonti scritte. Nel 1184 papa Lucio III scrive, infatti, all'abate Enrico di prendere sotto la protezione il monastero di San Severo. Oltre a fornire un elenco dettagliato dei beni del monastero, il papa dispone che il complesso di San Severo sia esente da decime e collette e concede, in tempo d'interdetto generale, la facoltà di officiare i servizi divini, il diritto di eleggere l'abate e la sepoltura libera ${ }^{28}$ : sono privilegi che mostrano la potenza e il prestigio di questo istituto anche rispetto agli altri monasteri ravennati.

Anche dopo la costruzione del monastero, il mausoleo mantiene il valore di spazio privilegiato e lo stesso avviene per l'area circostante. L'attività edilizia pertinente al complesso monastico ebbe effetto anche su questo piccolo edificio, che fu ulteriormente ampliato con l'aggiunta di un'abside poligonale allungata, orientata verso est. Questa modifica strutturale ne cambiò notevolmente la fisionomia, conformandola alla tipologia più diffusa di cappelle private a unica navata che nello stesso periodo si diffondono a Ravenna, come ad esempio S. Giustina in Capite Porticus, S. Giorgio de Porticibus e il piccolo edificio, la cui titolazione non è stata ancora identificata, rinvenuto nei pressi di via Antica Zecca ${ }^{29}$.

Sul versante occidentale del mausoleo, inoltre, le indagini archeologiche hanno identificato uno degli ingressi principali del monastero. Tramite questa entrata era possibile accedere agli ambienti interni, attraverso l'area porticata che si affacciava su di un lato sul chiostro. Dopo la fine del $\mathrm{X}$ secolo, questa zona di passaggio fu separata da quella di antica frequentazione cimiteriale, grazie alla costruzione di un recinto in muratura, che si impostava direttamente sull'abside del mausoleo originario. Associabili all'uso del recinto funerario, in linea con le fasi monastiche, sono state rinvenute alcune tombe collocate lungo il muro perimetrale ovest del complesso, destinate alla deposizione di più individui.

Nello stesso periodo, nell'area settentrionale della basilica, viene costruito un altro recinto funerario, utilizzato per separare l'area cimiteriale più antica, in fase con l'edificio religioso e formata da sepolture privilegiate, dalla zona che costeggiava la basilica e si estendeva davanti al nartece. Nell'area interna a questo recinto sono state rinvenute tombe in muratura, destinate ad accogliere esclusivamente sepolture femminili e singole, la cui distribuzione appare ordinata e precisa. Al di là del muro, invece, all'esterno del recinto, le sepolture si trovano in fosse terragne, in alcuni casi addossate le une alla altre, sia nei casi in cui gli individui sono stati deposti contemporaneamente sia in momenti differenti ${ }^{30}$.

In molti casi sono stati individuati dei laterizi, posti dietro la testa degli inumati, che sporgevano dal piano di calpestio dell'area cimiteriale, utilizzati come segnacoli. Si può ipotizzare, infatti, che parte di questi laterizi fosse visibile sia a coloro che frequentavano l'area cimiteriale, come parenti e devoti, nonché a coloro che svolgevano il ruolo di seppellitori, utilizzandoli come segnalazione utile nel momento in cui avveniva la deposizione di nuovi defunti, per mantenere così un'organizzazione cimiteriale più ordinata, evitando di intaccare le sepolture più antiche. Il cimitero posto in facciata della basilica era percorribile attraverso piccoli stradelli, che si aprivano all'interno di un giardino aperto, composto da graminacee selvatiche e arbusti di sambuco ${ }^{31}$, che molto probabilmente crescevano ai lati delle tombe (fig. 4).

\footnotetext{
${ }^{23}$ Per un più chiaro e completo inquadramento dei provvedimenti ecclesiastici carolingi si faccia riferimento a R. MCKITTERICK, Charlemagne. The Formation of a European Identity, Cambridge, 2008; M. DE JONG, Charlemagne’s Church, in J. Story (ed.), Charlemagne. Empire and Society, Manchester, 2005, p. 103-135.

${ }^{24}$ M. LAUWERS, Naissance du cimetière. Lieux sacrée et terre des morts dans l'Occident médiéval, Paris, 2005, p. 23-32.

${ }^{25}$ M. PIERPAOLI, Storia di Ravenna: compendio da Ottone III a Napoleone I: 1001-1805, Ravenna, 2001, p. 779.

${ }^{26}$ C. SAPIN, Dans l'église ou hors l'église, quel choix pour l'inhumé?, in H. Galinié, É. Zadora-Rio (eds.), Archéologie du Cimetière chrétien, Actes du $2^{\mathrm{e}}$ colloque ARCHEA, Tours, 1996, p. 68-69.

${ }^{27}$ R. GILCHRIST, B. SLOANE, op. cit. (nota 22).

${ }^{28}$ M. PIERPAOLI, op. cit. (nota 25), p. 771 n. 83.

${ }^{29}$ E. CIRELLI, op. cit. (nota 16).

3o Sul tema degli accessi e degli spazi monastici: E. DESTEFANIS, Ad portam monasterii. Accessi e spazi liminari nei monasteri dell'Occidente altomedievale (secoli -IX), in E. Destefanis, C. Lambert (a cura di), Per diversa temporum spatia. Scritti in onore di Gisella Cantino Wataghin, Torino, 2011, p. 51-84. ${ }^{31}$ Il paesaggio cimiteriale è stato ricostruito attraverso la combinazione dei dati archeologici e delle analisi paleobotaniche: M. L. CARRA, D. FERRERI, Analisi bioarcheologiche e attività funerarie presso la basilica di San Severo a Classe: l'area esterna al nartece, in Ocnus, 22, 2014, p. 81-9o. Sul paesaggio dei cimiteri si veda anche A. L. TROMBETTI BUDRIESI, Un gallo ad Asclepio: morte, morti e società tra antichità e prima età moderna, Bologna, 2013, p. 287.
} 


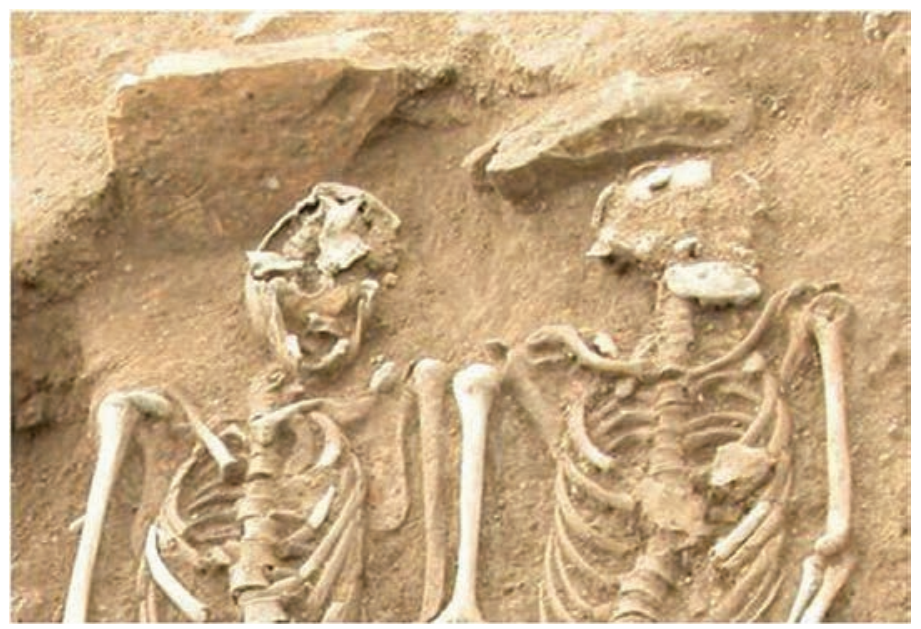

FIG. 4: Alcune sepolture con laterizi posti dietro la testa del defunto

L'utilizzo di questi segnacoli è attestato soltanto tra il X e il XII. La sparizione di tale pratica, nelle fasi più recenti, coincide con l'incremento del numero delle deposizioni e un'area cimiteriale invasa dalle sepolture. Tra il XIII e il XIV secolo, infatti, le sepolture sono posizionate all'interno di fosse terragne poco profonde evisibili. Gli inumati sono posti gli uni accanto agli altri, in maniera disordinata. Spesso le sepolture più antiche sono intaccate dalle deposizioni più recenti. Proprio per questo all'interno del deposito archeologico sono state individuate numerose ossa sparse e materiale

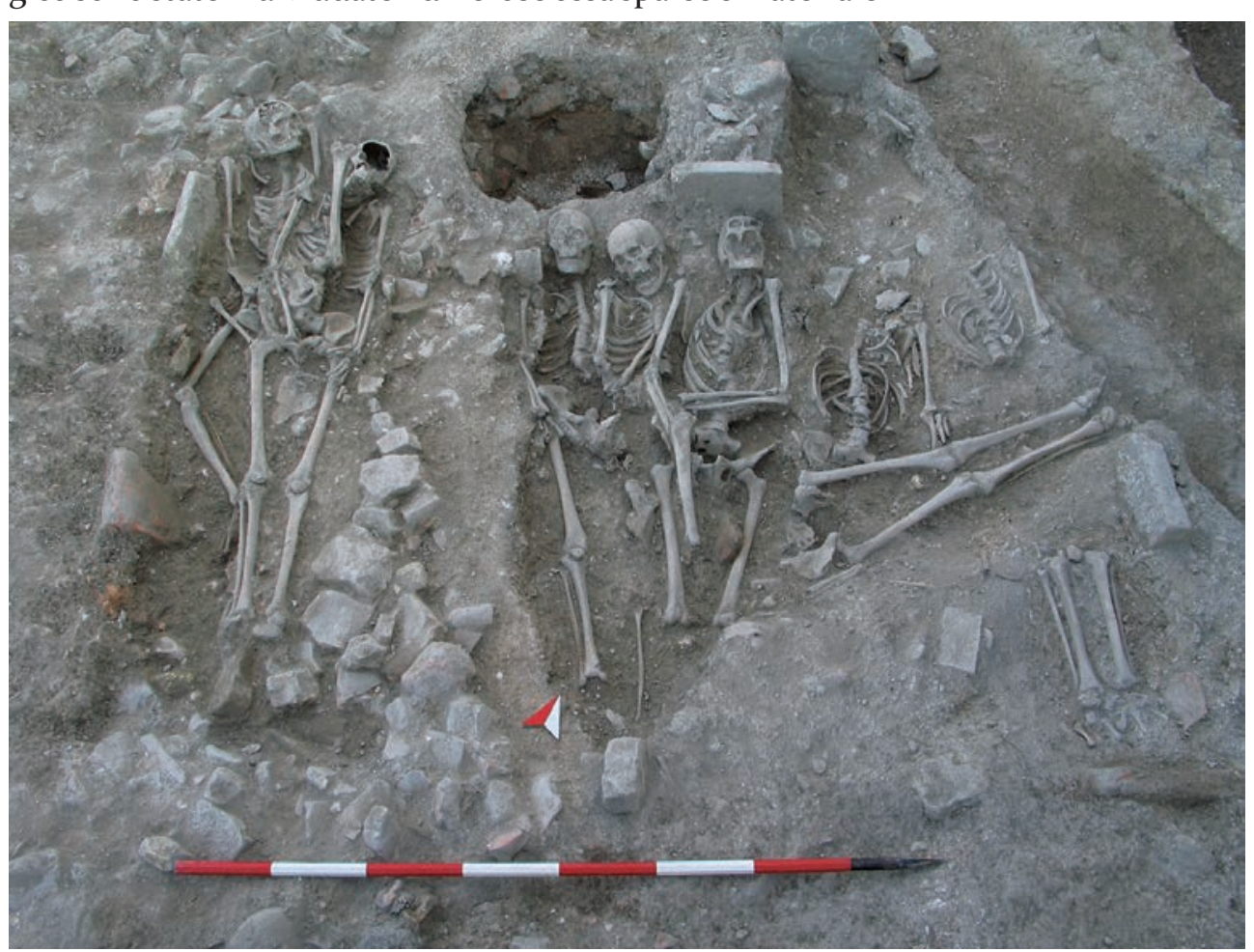

FIG.5: Sepolture nell'area esterna al nartece (XIII-XIV secolo) scheletrico disarticolato. La densità di sepolture è, in questi casi, tra la più elevata del complesso (fig. 5).

In rare occasioni i resti scheletrici delle sepolture disturbate sono preservate, con riduzioni mirate, magari accantonandole da un lato per far spazio alle nuove deposizioni ${ }^{2}$. Il campione antropologico è rappresentato da sepolture di entrambi i sessi, e di età diversificata, sebbene risalti notevolmente la fascia maschile di età adulta. Si può ipotizzare quindi che quest'area cimiteriale fosse destinata ai membri della comunità laica legata al complesso religioso, forse i familiari associati alle aristocrazie che sostenevano e guidavano il monastero. In taluni casi, l'osservazione di alcuni distretti scheletrici effettuate in corso di scavo, ha permesso di riconoscere l'utilizzo di un sudario per avvolgere il corpo del defunto prima della sepoltura ${ }^{33}$, nonostante non si siano conservate tracce di tessuto.

Infatti la deposizione del defunto all'interno di lenzuola, utilizzati come "contenitori", comprimeva il corpo, lasciando traccia di questa compressione nella posizione dello scheletro. Soprattutto a livello dell'articolazione della scapola e dell'omero, e la conseguente verticalizzazione delle clavicole ${ }^{34}$. Inoltre, in associazione a molti individui, sono stati rinvenuti diversi chiodi in ferro, localizzati intorno al cranio, ma del tutto assenti in prossimità di altre parti del corpo. In alcuni contesti cimiteriali di questi periodi la presenza di questi chiodi è stata associata a delle piccole assi di legno posti intorno alle teste dei defunti, in un tentativo di delimitazione delle singole sepolture, data la densità degli individui deposti nell'area ${ }^{35}$.

L'analisi della distribuzione delle tombe e il rapporto con il numero degli inumati ha rilevato una densità di sepolture minore all'interno degli spazi cenobitici. Probabilmente questo dato conferma la possibilità riservata solo ai monaci di essere seppelliti all'interno del complesso claustrale ${ }^{36}$. Le evidenze archeologiche hanno individuato, come possibili aree funerarie di pertinenza monastici, la sala capitolare, all'interno e nell'area esterna al perimetrale meridionale dell'ambiente, il cortile del chiostro e il portico colonnato che lo circondava.

Il chiostro, considerato il centro del monastero, è una delle aree più importanti del complesso. Intorno vi erano tre lati dell'edificio monastico e il lato meridionale della basilica. Architettonicamente è composto dalla parte centrale, ovvero il cortile, e da una zona di passaggio, il portico,

\footnotetext{
${ }^{32}$ La pratica di raccogliere e accantonare con cura le ossa delle deposizioni più antiche per deporre individui più recenti è invece identificata in altre aree del complesso. Nelle tombe rinvenute presso il mausoleo le ossa degli individui sono accantonate, in alcuni casi separando ossa lunghe da un lato e i crani dall'altro, per far spazio alle deposizioni più recenti.

${ }_{33}$ H. DUDAY, P. SELLIER, L'archèologie des gestes funèraries et la taphonomie, in Les Nouvelles De L’Archèlogie, 40, 1990, p. 15-18; H. DUDAY, Lezioni di archeotanatologia: archeologia funeraria e antropologia di campo, Roma, 2006.

${ }^{34}$ Tracce dell'utilizzo del sudario attraverso i resti scheletrici sono attestate in altri contesti monastici (R. GILCHRIST, B. SLOANE, op. cit. (nota 22); F. BERTOLDI, S. LORA, Archeologia funeraria e antropologia sul campo nel cimitero di San Lorenzo, in F. Bertoldi, M. Librenti M. (a cura di), Nonantola 2. Il cimitero bassomedievale della chiesa di San Lorenzo nel Borgo di Nonantola, Firenze, 2007, p. 19-34.

35 R. GILCHRIST, B. SLOANE, op. cit. (nota 22).

${ }^{36}$ C. SAPIN, op. cit. (nota 10), p. 68-69.
} 


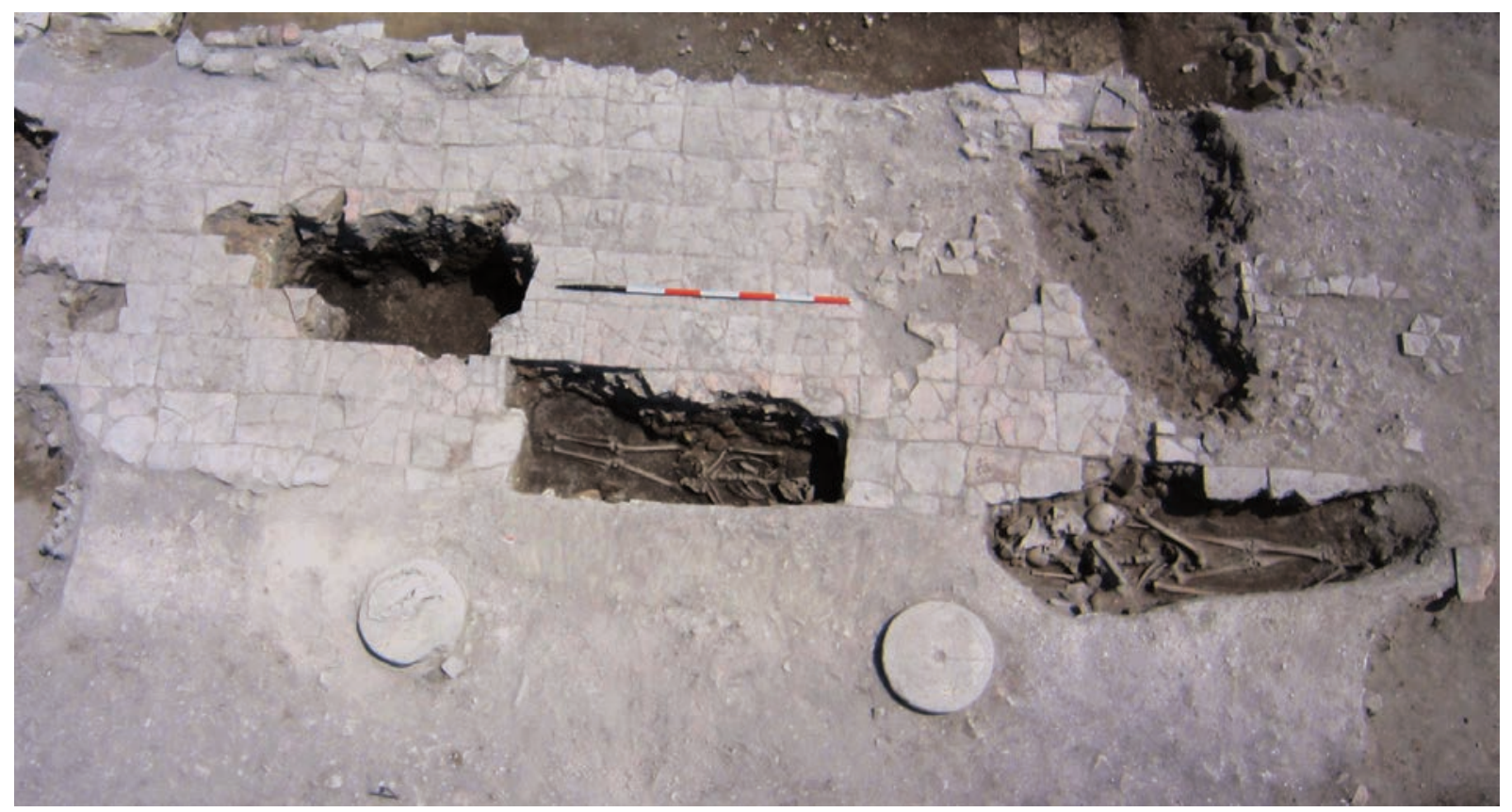

FIG. 6: Sepolture all'interno del portico del chiostro.

che correva su tre lati, interamente pavimentato in laterizi. Attraverso questi passaggi si poteva accedere alla basilica, sul lato della navata meridionale, e ad alcuni ambienti del monastero. All'interno della pavimentazione del porticato che circonda il chiostro sono state rinvenute diverse sepolture, probabilmente riferibili alla comunità monastica: sul lato più orientale ne sono state rinvenute due, vicine tra loro, e orientate nord-sud. Gli inumati sono all'interno di fosse molto profonde, tagliate nel pavimento del portico. Dalla profondità e dal tipo di decomposizione dei resti scheletrici si è ipotizzata una copertura marmorea al di sopra di queste tombe, tale da renderne la presenza visibile quando i monaci percorrevano il portico.

Probabilmente le lastre sono state portate via insieme agli altri elementi marmorei del portico, come le colonne, nell'attività di spoliazione che ha interessato l'intero complesso. Queste sepolture sono databili a una fase subito posteriore all'XI secolo (fig. 6). Un altro gruppo di sepolture è localizzato all'interno del portico, sul versante occidentale, vicino alla basilica. Le tombe sono orientate nord-sud, come il muro del portico duecentesco. Si tratta di sepolture in fossa terragna, ma in questo caso di non notevole profondità. All'interno del cortile del chiostro, invece, sono stati riportati alla luce solo alcuni ossari. La zona era molto probabilmente tenuta a giardino, con aiuole separate da vialetti, in ciottoli e ghiaia pressati, le cui tracce sono state trovate soprattutto nella zona centrale, leggermente spostato verso est, dove era posizionato un pozzo, decorato con blocchi marmorei scolpiti e caratterizzati da un motivo di foglie e girali di vite, rinvenuto solo in parte perché in gran parte asportato alla fine del XV secolo ${ }^{37}$.

Sul versante opposto rispetto al pozzo, nella parte meridionale, era presente una fontana, costruita con materiale di recupero, come un capitello e una transenna in marmo, utilizzato come scolina per l'acqua, aggiunti però nel XIII secolo. In quest'area non sono state rinvenute sepolture in posizione primaria, ma solo secondarie, all'interno di fosse circolari. Non possiamo escludere però che la posizione di questi individui fosse precedentemente all'interno di sarcofagi o di altre tipologie di sepoltura più stabili, forse originariamente all'intero del chiostro e in seguito ai cambiamenti di funzione, rimosse e deposte nei piccoli ossari. Alcune fosse di funzione indeterminata, sono state ad esempio rinvenute nel portico aggiunto nel XIII secolo, in collegamento tra basilica e monastero. Sebbene prive di resti scheletrici, la forma e la profondità delle fosse fanno ipotizzare una loro funzione sepolcrale ${ }^{38}$. L'analisi antropologica su questi resti scheletrici ha riscontrato la presenza di infanti e di adolescenti. Si tratta forse di oblati, affidati da parte dei genitori in giovane età ai monasteri39.

\footnotetext{
37 A. AUGENTI et alii, op. cit. (nota 12).

${ }^{38}$ In altri monasteri italiani, come ad esempio, San Michele alla Verruca, il chiostro viene utilizzato come area di sepoltura, ma solo in una fase circoscritta (S. GELICHI, A. ALBERTI, M. DADÀ, L'indagine archeologica del monastero di San Michele alla Verruca: la periodizzazione della sequenza insediativa, in S. Gelichi, A. Alberti (a cura di), L'aratro e il calamo. Benedettini e Cistercensi sul Monte Pisano. Dieci anni di archeologia a San Michele alla Verruca, Pisa, 2005, p. 63-127). A Brescia, invece, nel monastero di San Salvatore, la più ampia area cimiteriale del complesso si è sviluppata proprio nel cortile del chiostro centrale, posto a sud della chiesa, dove sono state rinvenute un centinaio di sepolture, di cui 55 a cassa (G.P. BROGIOLO, Sepolture privilegiate altomedievali nel monastero di S. Salvatore di Brescia, in Hortus Artium Medievalium, 10, 2004, p. 15-24; G. P. Brogiolo, F. Morandini (a cura di), Dalla corte regia al monastero di San Salvatore-Santa Giulia di Brescia, Mantova, 2014). Nel monastero di Cairate sono state rinvenute più di 200 sepolture, cronologicamente distribuite lungo l'intera vita del complesso. All'interno del chiostro ne sono state rinvenute soltanto 3 in cassa (M. MOTTO, Sepolture nel monastero di Cairate: tipologia e organizzazione delle aree cimiteriali, uno sguardo di sintesi, in V. Mariotti (a cura di), Un monastero nei secoli. Santa Maria Assunta di Cairate, scavi e ricerche, Mantova, 2014, p. 501-517). Il chiostro di San Vincenzo al Volturno, circondato da un portico di colonne e la parte centrale decorata con un giardino, non ha restituito nessuna area cimiteriale. Le sepolture dei monaci sono state riconosciute negli inumati rinvenuti all'interno dell'atrio della basilica (per lo studio delle sepolture e dei resti scheletrici V. HIGGINGS, The people of San Vincenzo in Late Antiquity and the Early Middle Ages, in J. Mitchell, L.I. Hansen (eds), San Vincenzo al Volturno 3: the finds from the 1980-86 excavations, Spoleto, 2001, p. 425-437).

39 S. MAYS, The osteology of Monasticism in Medieval England, in R. Gowland, C. Knüsel (eds.), Social archeology of funerary remains, Oxford, 2006, p. 179-189; C.H. LAWRENCE, Medieval Monasticism, London, 1984, p. 32; sulla presenza dei bambini oblati, G. ARCHETTI, «Sub virga magistri». Custodia e disciplina nell'educazione carolingia dei pueri oblati, in Studi medievali, LVII, 2 (2016), p. 527-582.
} 


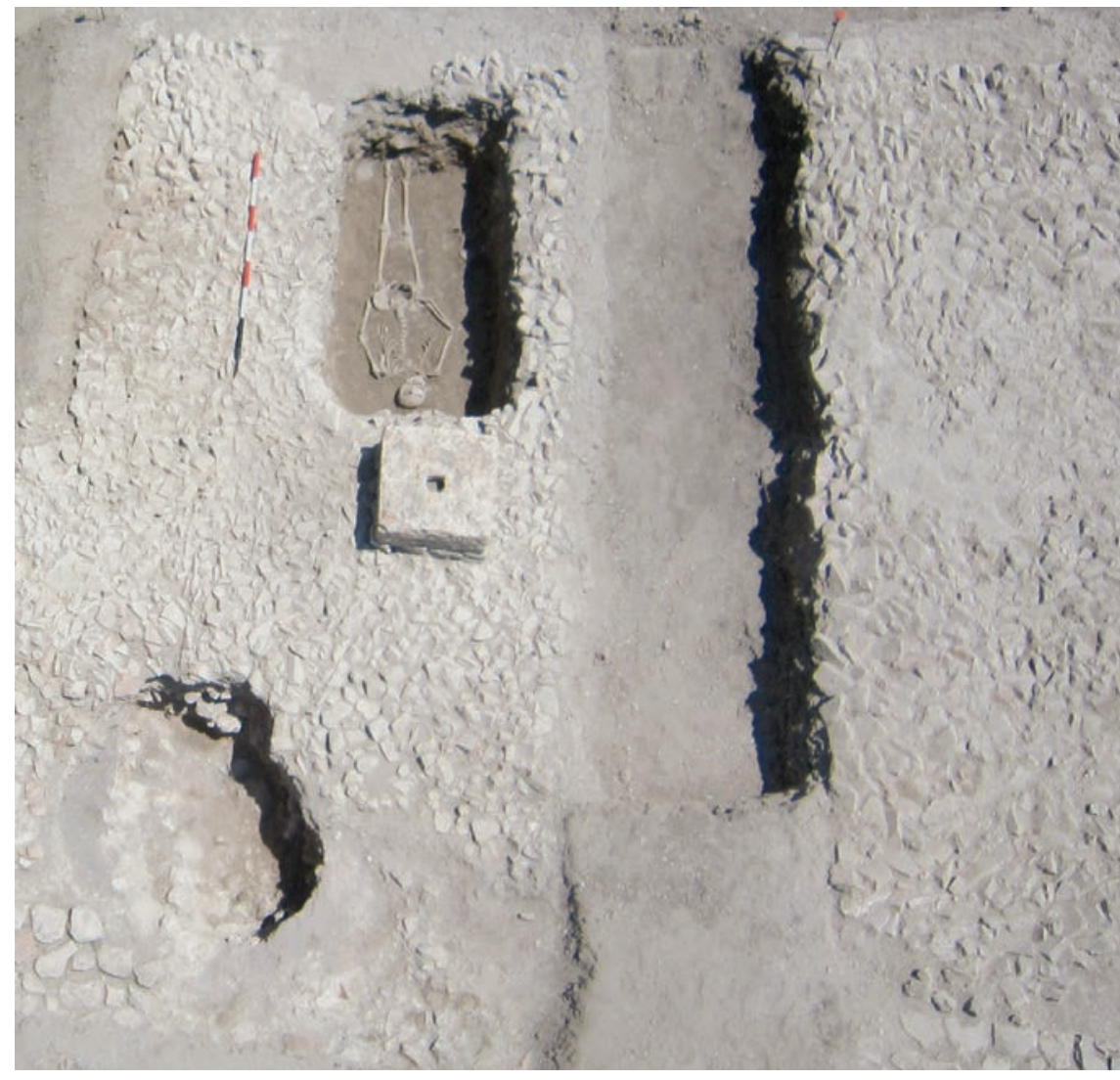

FIG. 7: Sepoltura rinvenuta all'interno della sala capitolare

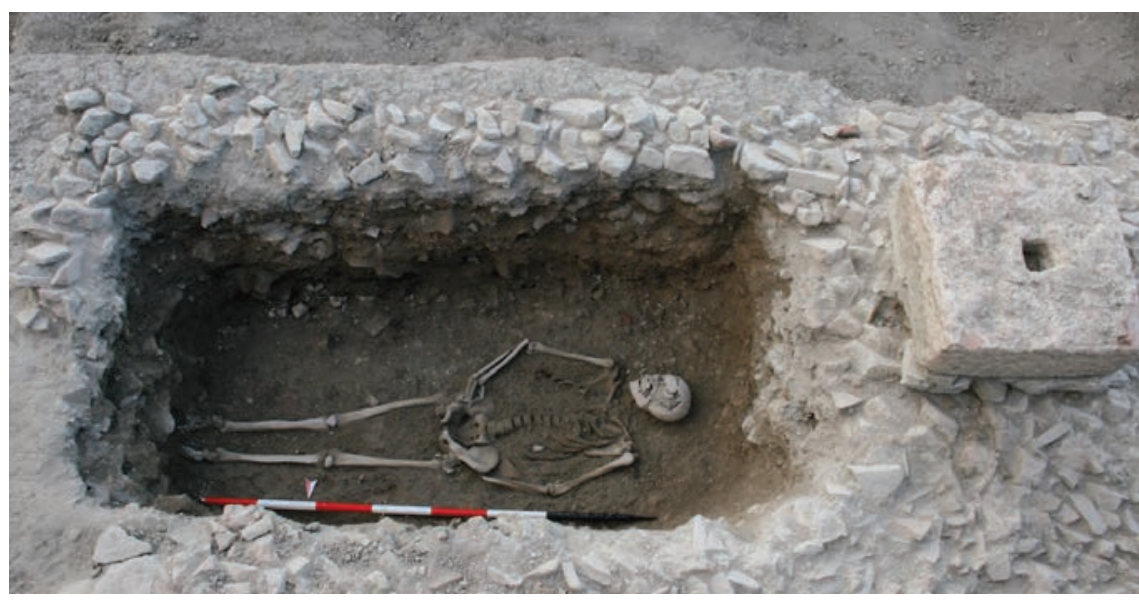

FIG. 8: Sala capitolare, sepoltura con a lato blocco in marmo.
All'interno della sala capitolare di San Severo è stata rinvenuta una sola tomba, databile intorno alla metà del XIII secolo, notevole per dimensioni ma anche per la sua posizione, nella zona più occidentale dell'ambiente, messa in risalto dalla presenza di un blocco quadrangolare in marmo rosso di Verona sul quale vi era collocata una colonna, o un segnacolo monumentale, in base al perno presente al centro del blocco ${ }^{40}$ (fig. 7) La sepoltura ha un orientamento est-ovest. L'inumato era collocato all'interno di una fossa molto profonda e larga, probabilmente coperta da una lastra marmorea. La sepoltura appartiene a un uomo adulto, la cui età al momento della morte è di circa 40 anni, di statura elevata, all'incirca 1,8o m (fig. 8).

Si tratta molto probabilmente di uno degli abati del monastero, vissuti nel momento di passaggio della comunità dall'ordine benedettino a quello cistercense, avvenuta a metà del XIII secolo, sebbene non si conosca con precisione l'anno esatto di questo cambiamento ${ }^{41}$ e tantomeno la lista completa degli abati del monastero.

Nel lato più occidentale della sala capitolare è stato rinvenuto anche un ossario. La pratica di seppellire all'interno dell'ambiente capitolare o nelle dirette vicinanze è prassi comune, presso molti impianti monastici, sia in Italia ${ }^{42}$ sia nel resto d'Europa ${ }^{43}$. Per il valore simbolico e prestigioso era nella maggior parte dei casi un luogo destinato alla sepoltura dei monaci ${ }^{44}$. In alcuni casi in questo ambiente potevano trovare sepoltura anche i laici, ma solo in casi eccezionali con personaggi di particolare importanza ${ }^{45}$. Le prime sepolture ${ }^{46}$ all'interno della sala capitolare sono attestate già a partire dall'XI secolo ${ }^{47}$. Nella sala capitolare di Durham CathedralPriory, sono attestate, tra il 1128 e il 1316, le sepolture di cinque vescovi. Gli abati di Byland Abbey, un altro monastero nello Yorkshire, sono seppelliti nella sala capitolare tra il XII e il XIII secolo; e allo stesso periodo appartengono le sepolture di Bury St Edmunds ${ }^{4}$.

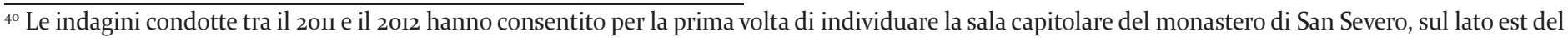
chiostro, in uno spazio compreso tra l'esterno della navata meridionale della basilica, l'abside e l'esterno del muro perimetrale del complesso ecclesiastico. ${ }^{41} \mathrm{~J}$. LASZLOVSKY, The monastery of St. Severo in Classe, Ravenna, in Annual of Medieval Studies at CEU, 13, 2007, p. 197-211; B. BEDINI, Le abazie cistercensi d'Italia (sec. XII-XIV), Roma, 1964.

${ }^{42}$ F. SBARRA, Il cimitero: cronologia, organizzazione e aspetti della ritualità, in S. Gelichi, A. Alberti (a cura di), L'aratro e il calamo. Benedettini e Cistercensi sul Monte Pisano. Dieci anni di archeologia a San Michele alla Verruca, Pisa, 2005, p. 223-224.

${ }^{43}$ In altri monasteri le prime sepolture all'interno della sala capitolare sono attestate già a partire dall'XI secolo: N. ROGERS, Monument to monk and monastic servant, in B. Thompson (ed.), Monasteries and society in medieval England, Nottingham, 1999 (Harlaxton Medieval Studies, 6), p. 273-726.

${ }^{44}$ G. COPPACK, Abbeys and Priories, London-Batsford, 1990, p. 72; N. COLDSTREAM, Cistercian architecture from Beaulieu to the dissolution, in C. Norton, D. Park (eds.), Cistercian art and architecture in the British Isles, Cambridge, 1986, p. 139-159.

${ }^{45}$ C. DANIELL, Death And Burial in Medieval England: 1066-1550, London-New York, 1977.

${ }^{46}$ All'interno è stato identificato un solido piano in malta, probabilmente la preparazione di un pavimento in marmo di XI secolo, rialzato nel corso del $\mathrm{XIV}$, di cui sono stati rinvenuti numerosi frammenti negli strati di abbandono e rialzamento successivi. I materiali rinvenuti all'interno hanno permesso inoltre di ipotizzare una decorazione di pregio sulle pareti, anche con tessere musive in pasta vitrea rivestite da foglie dorate, sottoposte ad analisi archeometriche che ne confermano la cronologia e mostrano una attività di produzione di questo materiale forse proprio all'interno del monastero (M. VANDINI, R. ARLETTI, E. CIRELLI, Five centuries of mosaic glass at Saint Severous, in Ocnus, 22, 2014, p. 91-108). Associabile ad essa è stata individuata, sul lato orientale della sala, una struttura rialzata in muratura, interpretata come una probabile tribuna, utilizzata dai monaci durante la riunione del capitolo.

${ }^{47}$ N. ROGERS, Monument to monk and monastic servant, p. 273-726.

${ }^{48}$ R. GILCHRIST, B. SLOANE, op. cit. (nota 22), p. 59.
} 
Dalle analisi antropologiche effettuate su alcuni contesti, sembra che nella maggior parte dei casi fosse destinata alla sepoltura di soli maschi adulti, come i monasteri di Ipswich Blackfriars ${ }^{49}$, Ipswich Whitefriars ${ }^{50}$, Gilbertine Priority of St Andrew, Fishergate, York ${ }^{51}$, St Gregory'sPriory of Augustinian canons e Canterbury ${ }^{52}$, in cui sono state riconosciute come sepolture di membri della comunità religiosa. È rilevante segnalare, però, alcuni casi in cui nella sala capitolare sono state rinvenute sepolture di sesso femminile, come a MertonPriory ${ }^{33}$ e LinlithgowWhitefriars ${ }^{54}$, e di bambini, come a Oxford Blackfriars ${ }^{55}$.

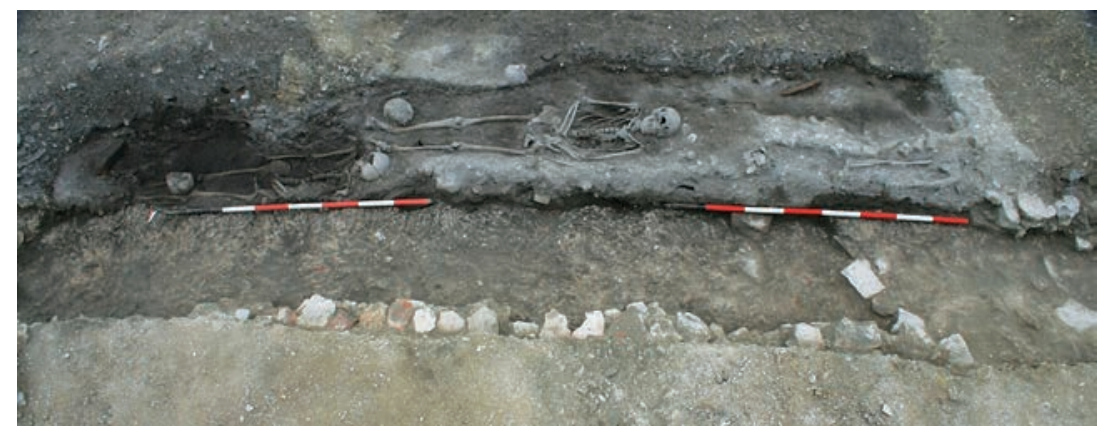

FIG.9: Sepolture esterne alla sala capitolare, addossate al muro perimetrale.

All'esterno della sala capitolare, sul versante meridionale dell'ambiente, la pratica funeraria è ascrivibile a due momenti distinti di vita del monastero. La più recente, che corrisponde anche a un utilizzo più intenso, è compresa tra il XIV e il XV secolo. Si tratta dell'ultima fase di vita del monastero, quando questa zona del complesso era utilizzata come area cimiteriale nella porzione più settentrionale, e per attività produttive nella porzione più meridionale, come indica il rinvenimento di una fornace per la lavorazione del bronzo, posta al di sotto di una copertura lignea. Le fosse sepolcrali sono ricavate all'interno di un piano in malta, riconoscibile come il piano di calpestio dell'area. Le tombe sono disposte lungo il muro perimetrale della sala capitolare, all'interno di una fossa parallela all'ambiente. Gli individui sono orientati con la testa verso ovest; in alcuni casi è presente un laterizio utilizzato come cuscino funerario. Sono presenti alcuni laterizi lungo le pareti della fossa, non legati tra di loro, ma in modo discontinuo senza creare delle vere e proprie murature.

Una volta asportate queste sepolture e il piano in malta è stata individuata un'ulteriore fase cimiteriale, compresa tra fine XI eXII secolo. Gli inumati continuano a essere disposti con il capo orientato verso ovest, come le precedenti, e sono posizionate sempre parallele al muro della sala capitolare, sebbene a una certa distanza. Gli individui sono all'interno di fosse terragne, prive di qualsiasi elemento in laterizio. L'area cimiteriale era divisa dalla zona artigianale probabilmente da una piccola struttura muraria, ma gli spazi erano a contatto diretto (fig. 9).

Un' altra zona dal forte valore privilegiato, e probabilmente utilizzata dal clero e dalla comunità monastica, è quello esterno all'abside, dove sono state rinvenute alcune tombe in muratura, addossate all'esterno della struttura absidale. Le tombe, grandi e monumentali, sono cronologicamente associabili alle prime fasi della basilica, fine VI secolo, e utilizzate fino alle fasi monastiche più recenti. Sono stati rinvenuti i resti di scheletrici di soli uomini, giovani e anziani ${ }^{5}$.

Le analisi antropologiche effettuate sui resti scheletrici hanno evidenziato che gli spazi funerari prettamente legati al monastero sono tutti destinati a uomini, sia giovani sia adulti (e ovviamente ciò non stupisce considerando che si tratta di un monastero maschile). Per quanto riguarda le patologie del nostro campione, le più diffuse sono quelle articolari. Le malattie infettive, invece, sono scarse. In questi casi il distretto scheletrico più interessato è quello degli arti inferiori, soprattutto tibie e fibule. A carico degli arti inferiori è la maggior parte degli stress funzionali riscontrati. È stata rilevata, infatti, una presenza elevata di squatting facets dell'epifisi distale della tibia, risultati da probabile e ricorrente flessione dell'astragalo sulla caviglia ${ }^{57}$. Questi indicatori denotano una frequente assunzione della posizione accovacciata o di quella seduta su sedili bassi. Inoltre i segni riconducibili ad attività fisiche che prevedono piegamenti continui delle gambe e posizioni accovacciate sono state identificate a livello delle ossa dei piedi.

Abbastanza frequenti sono le infezioni a livello del periodonto, legati ai processi infiammatori che colpiscono le gengive, a causa del deposito della placca batterica, dovuta solitamente a una scarsa igiene orale e il consumo di carboidrati. Inoltre, sono attestate numerosi casi di perdite di

\footnotetext{
${ }^{49}$ S. MAYS, The Medieval Burials from the Blackfriars Friary, School Street, Ipswich, Suffolk (excavated 1983-85), Part I\&II, Ancient Monuments Laboratory Report 16/91, London, 1991.

${ }^{50}$ S. MAYS, The Burials from the Whitefriars Friary Site, Buttermarket, Ipswich, Suffolk (excavated 1986-88), Ancient Monuments Laboratory Report 17/91, London, 1991.

${ }^{51}$ G. STROUD, R. L. KEMP, Cemeteries of St Andrew, Fishergate, The Archaeology of York 12/2, York, 1993.

${ }^{52}$ T. ANDERSON, The human remains, in M. Hicks, A. Hicks (eds.), St Gregory's Priory, Northgate, Canterbury Excavations 1988-1991, Canterbury, 2001, p. 338-370.

53 T. WALDRON, A report on the Human Bone from Merton Priory, Ancient Monuments Laboratory Report 4483, London, 1985.

${ }^{54}$ J. A. STONES, Three Scottish Carmelite Friaries. Excavation at Aberdeen, Linlithgow and Perth 1980-1986, Society of Antiquaries of Scotland Monograph 6, Edinburgh, 1989.

${ }^{55}$ E. EDWARDS, The human remains, in G. Lambrick, H. Woods (eds.), Excavation at the Second Site of the Dominican Priory, Oxford, 1976 (Oxoniensia, 41), p. 168-231.

${ }^{56}$ Queste sepolture sono state riportate alla luce nel corso delle indagini che hanno interessato la basilica nel corso degli anni '6o, non è possibile quindi avere una cronologia più dettagliata, per quanto riguarda lo studio antropologico effettuato sui resti scheletrici si rimanda a F. MARTUZZI VERONESI, G. MALACARNE, Note antropologiche su reperti romani e medioevali del territorio di Classe (Ravenna), in Archivio per l'Antropologia e la Etnologia, 98, 1968, p. 147-164.

${ }^{57}$ E. L. BOULLE, Evolution of two human skeletal markers of the squatting position: a diachronic study from antiquity to the modern age, in American Journal of Physical Anthropology, 115, 2001, p. 50-56; L. CAPASSO, I fuggiaschi di Ercolano. Paleobiologia delle vittime dell'eruzione vesuviana del 79 d.C., Roma, 2001, p. 145; A. CANCI, S. MINOZZI, Acheologia dei resti umani. Dallo scavo al laboratorio, Roma, 2005.
} 


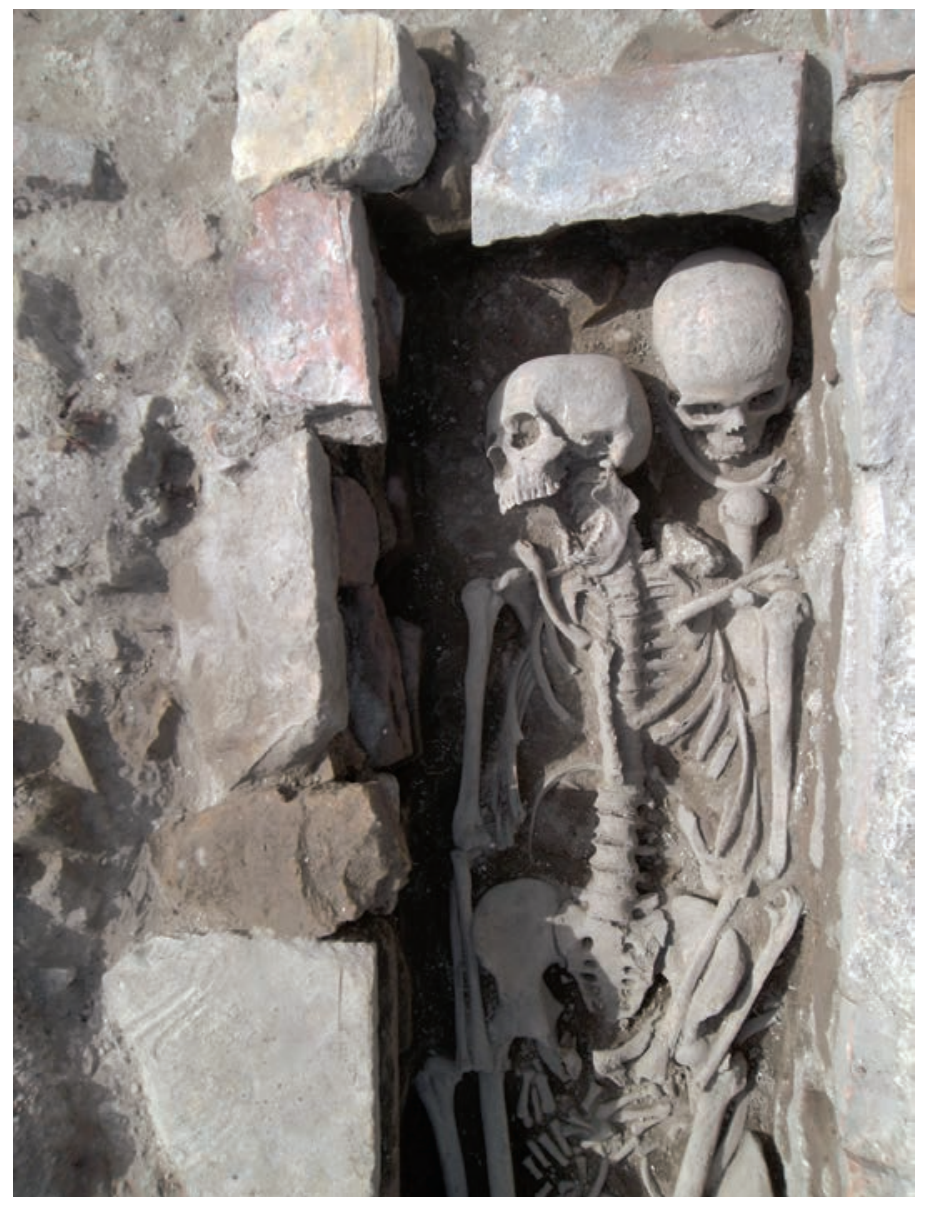

FIG. 10: Sepoltura bisoma presso uno degli ingressi del monastero.

denti in vita. Tra le cause più ricorrenti vi sono la forte usura delle superfici occlusali, affezioni del periodonto, carie, fattori genetici e l'età avanzata. Sono presenti anche i segni di un uso prolungato dei muscoli masticatori, evidenziando una probabile attività masticatoria extra alimentare, legate all'attività di tessitura o alla tiratura del cordame per le reti da pesca ${ }^{58}$.

La maggior parte delle sepolture rinvenute all'interno dell'intero complesso sono singole, ma numerosi sono i casi di sepolture multiple, bisome o di sepolture secondarie. Alcune di queste deposizioni avvenivano simultaneamente, come alcune tombe bisome rinvenute sia all'interno del mausoleo sia nell'area esterna al nartece, altre in momenti differenti (fig. 10). In questi casi le tombe, in particolar modo quelle grandi in muratura, potevano accogliere fino a dieci defunti (fig. 11).

Quando le tombe venivano aperte per la collocazione dei corpi dei defunti, i resti delle sepolture più antiche rimanevano all'interno della stessa tomba ma, essendo completamente disarticolate, si accantonano, con cura, riducendo lo spazio occupato, per far posto ai defunti più recenti59. L'atto di spostare le ossa di defunti più antichi da parte dei seppellitori è svolto con attenzione nei confronti dell'individuo, sebbene la manipolazione delle ossa, le riduzione dei resti scheletrici, provocava la perdita delle connessioni anatomiche degli individui, il mescolamento tra di esse, e la

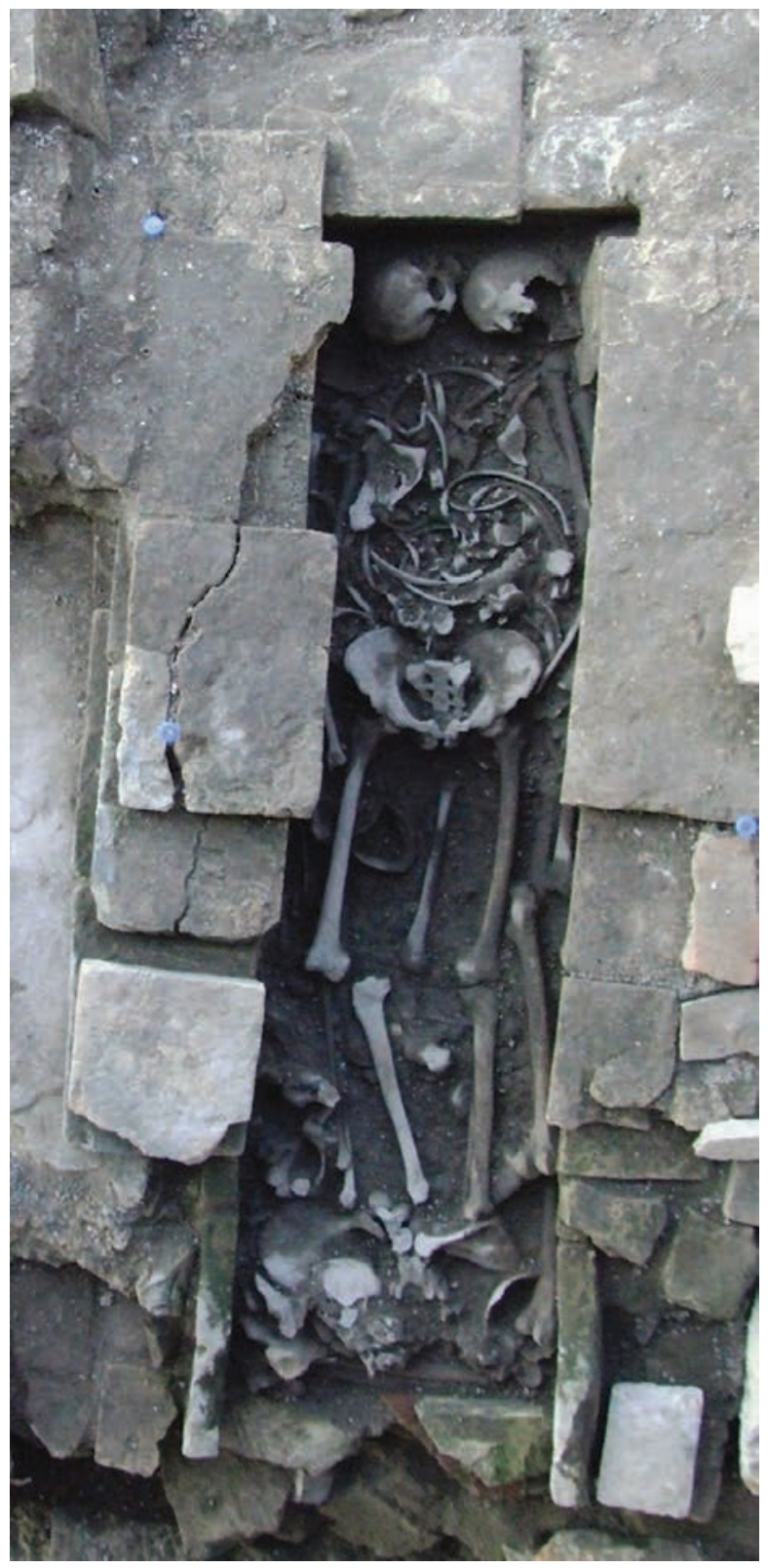

FIG.11: Tomba in muratura con sepolture multiple e riduzioni.

perdita della loro individualità. Questo poteva avvenire sia nel caso di una diretta relazione familiare tra i defunti, sia tra sepolture legate dall'appartenenza alla stessa comunità. Per questo in alcune sepolture si è notata una particolare attenzione verso i crani, collocati spesso accanto al capo dei defunti recenti, in una posizione più rilevante.

In questo atteggiamento nei confronti dei corpi dei defunti e nella conservazione delle tombe antiche emerge come la priorità del luogo prevalga invece sull'individualità della sepoltura. In questo modo viene superato un tabù antico, presente ancora nell'alto Medioevo, verso le profanazioni delle tombe, la loro riapertura, anche nei casi in cui i parenti volessero deporvi altri cari. In questi gesti di spostamento di ossa e sovrapposizione di defunti è importante la vicinanza presso i defunti più antichi e il mantenimento della posizione all'interno della tomba. L'importanza di essere seppelliti

\footnotetext{
$5^{8}$ E. CIRELLI, La dieta dei monaci. Cultura materiale e alimentazione nei monasteri benedettini tra IX e X secolo, in Hortus Artium Medievalium, 19, 2013, p. 227-240.

${ }^{59}$ La presenza di sepolture secondarie indica una lunga e intensa attività funeraria dei contesti cimiteriali, attestata in altri cimiteri monastici: S. BULLY, M. JURKOVIĆ, M. ČAUŜEVIĆ-BULLY, I. MARIĆ, Le monastère Saint-Pierre d'Osor (Île de Cres). Neuvième campagne d'études archéologiques, in Hortus Artium Medievalium, 21, 2015, p. 356-365.
} 
vicino agli avi, è soprattutto legata al mantenimento dello stesso luogo. Il cimitero, spazio consacrato, può essere riusato e modificato, come avviene con le ossa delle tombe più antiche, che vengono rimaneggiate, spostate e accantonate per far spazio ad altri corpi.

Nel corso del tempo il rapporto con la morte e la pratica funeraria della comunità ravennate evolvono attraverso una compresenza di identità e di rappresentazioni sociali. Il culto memoriale del vescovo Severo attrae le sepolture sia attorno al mausoleo, sia nell'area della grande basilica. La disposizione delle sepolture non si limita a seguire le tradizionali dinamiche delle sepolture ad sanctos ma evidenzia sviluppi cimiteriali in cui le tombe privilegiate sono quelle che mantengono la propria collocazione nel corso del tempo.
Queste tombe sono riutilizzate nel corso di più generazioni e conservano i legami familiari.

Alla comunità monastica di San Severo è affidata la cura e la custodia di uno degli edifici di culto più importanti dell'area, tutelando e mantenendo il luogo della sepoltura del santo, presso una zona dotata di tradizione a partire dall'epoca romana. I membri della comunità di Classe continueranno a seppellire i propri defunti presso questo luogo, parte rilevante della storia di questo territorio e in cui si esprime il valore della memoria e dell'identità della città, fino a quando il complesso, intorno alla metà del XV secolo, sarà completamente abbandonato e diventerà una cava a cielo aperto di materiali da costruzione ${ }^{60}$.

\footnotetext{
${ }^{60}$ Il 6 dicembre 1455 il monastero di San Severo, per volere di Callisto III fu accorpato a quello di Sant'Apollinare in Classe e sul luogo della memoria del vescovo rimase solamente una piccola chiesa, demolita definitivamente agli inizi del XIX secolo, cfr. R. Benericetti (a cura di), Le carte ravennati del secolo decimo. Archivi minori (monasteri di Sant'Andrea Maggiore, San Vitale e Sant'Apollinare in Classe), Faenza, 2006.
} 